\title{
Further Exploration Of Global Asset GMVPs: Does Risk Reduction Benefit From Weekly Data?
}

Arben Zibri, University of New York Tirana (UNYT), Albania

Agim Kukeli, Black Hills State University, USA

\begin{abstract}
The paper studies the differences in risk reduction among global minimum variance portfolios (GMVPs) derived from the optimization of weekly and monthly return. This research follows the analysis of Zibri and Kukeli (2014) regarding differences in performance which is measured for different revision frequencies and transaction costs. The data used are weekly/monthly returns for stock, bond, gold, oil indices for the period 1996-2013. If more frequently rebalancing is applied, most restrictive weekly GMVPs reduce risk more than returns vis-à-vis monthly GMVPs.
\end{abstract}

Keywords: Portfolio Optimization; Minimum Variance Performance; Global Assets; Return Data Frequency

\section{INTRODUCTION}

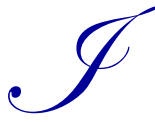

nspired by the work of Jaganathan and Ma (2003), this research advances on the study of Zibri and Kukeli (2014) on the difference in out-of-sample risk reduction of Global Minimum Variance Portfolios (GMVPs) constructed using sample covariance matrices of monthly returns and weekly returns.

Jagannathan and Ma (2003) find that sample covariance matrix and shrinkage estimators of daily returns perform the best based on risk reduction. Constraints, such as no-short sale and maximum bound, do not help performance improve as the data frequency increases from monthly to daily, according to the authors.

As per Gosier et al. (2005), the estimated volatility using daily returns is lower than the volatility using monthly returns. Daily GMVPs beat monthly GMVPs in terms of volatility independently from the forecasting model for the covariance matrix used.

Clarke et al. (2006) uses past year daily return $(T=250)$ and past five years monthly return $(T=60)$ to estimate the variance covariance matrix of returns for the 1,000 large market capitalization stocks in the U.S. markets, through a shrinkage estimation process. They find that GMVPs estimated by daily data show risk-return improvement compared to monthly GMVPs.

Applying a similar methodology of Behr et al. (2008) and Jagannathan and Ma (2003), this research compares the risk reduction characteristics of minimum variance portfolios of global asset classes estimated by different input data frequency. Global assets are represented by country and/or regional indices of stocks, corporate and government bonds, precious metals, and energy. The authors use the monthly and weekly returns of the above mentioned indices. Following the conclusions of Pantaleo et al. (2011) ${ }^{1}$, this paper uses the sample covariance matrix as estimator of the covariance matrix of returns. The short sale/upper bound optimization restrictions suggested by Behr et al. (2008) are considered in this research.

\footnotetext{
${ }^{1}$ Referring to the empirical study of the authors, if the number of observations, $\mathrm{T}$, is greater than the number of assets, $\mathrm{N}$, improved estimators of the covariance matrix of returns do not perform better than sample covariance matrix out-of-sample in terms of risk reduction.
} 
The main results of this research are:

1. Unconstrained and no-short sale restricted global minimum variance portfolios experience lower out-ofsample standard deviation if monthly returns are used in the estimation of the sample covariance matrix.

2. Upper/lower bound constrained GMVPs perform the best, based on risk reduction, when derived from weekly historical returns.

3. Less frequent rebalancing policy penalizes GMVPs estimated using higher frequency data, referring to the findings of this research.

Subsequent sections present the methodology of portfolio construction and optimization, introduce the data used in this research, analyze the empirical findings of how changes in data frequency impact performance GMVPs vis-à-vis benchmarks and how this is affected by the portfolio revision frequency, and summarize conclusions and recommendations.

\section{RESEARCH METHODOLOGY}

The methodology of this paper closely follows the one used from Behr et al. (2008). The returns are presented in EURO currency. This section is divided in two parts.

The first part describes the variance-covariance matrix estimation approach and optimization, and the second part briefly explains the inclusion of transaction costs.

\section{Portfolio Optimization}

Following Markowitz's (1952) portfolio selection framework, this paper employees the global minimum variance portfolio. The variance-covariance matrix estimation period is a tradeoff between statistical confidence and possibility of inclusion of irrelevant data. In line with Chan et al. (1999) and Jagannathan et al. (2003), this work uses an estimation window of 60 months and 52 weeks for the construction of sample covariance matrix for the respective monthly and weekly GMVPs. Optimization is performed on a rolling sample basis with one-year and three-year revision frequency.

The traditional estimator for the sample covariance matrix $\mathrm{S}$ is given by the formula:

$S=\frac{1}{T-1} \sum_{t=1}^{T}(R t-\bar{R})(R t-\bar{R})^{\prime}$

$\mathrm{T}$ is the sample size, $R t$ is the $\mathrm{N}^{\mathrm{x}} 1$ vector of asset return at time $\mathrm{t}, \bar{R}$ represents the sample means of these returns, and $\mathrm{N}$ represents the number of assets considered in the portfolio. Given the estimated sample covariance matrix, the Global Minimum Variance Portfolio (GMVP) is the solution of the following optimization problem:

$$
\begin{aligned}
& \min _{W \in R} W^{\prime} \sum^{\wedge} W \\
& \text { s.t. } \sum_{i=1}^{n} W i, t=1
\end{aligned}
$$

Wi,t denotes the weight of asset $\mathrm{i}$ at period t. The constraint in equation 3 implies that total portfolio weight, including short positions, should sum up to 1 . This is the classical unconstrained minimum variance portfolio.

The solution to minimum variance optimization often is empirically a few assets with extreme long and short positions. Jagannathan and Ma (2003) find the shrinkage effect of the upper/lower bound restrictions presented in equations 4 and 5.

$$
W i, t \geq 0, \text { for } i=1 \ldots n
$$




$$
W i, t \leq W^{\max }, \text { for } i=1 \ldots n
$$

This research studies the difference in risk reduction characteristics of three minimum variance portfolios: 1) the unconstrained, 2) the no-short sale constrained, and 3) the no-short sale and upper bound constrained for global asset classes. Weekly returns, monthly returns and respective sample covariance estimates are used as input for GMVP optimization.

\section{Transaction Costs}

Transaction costs in the portfolio returns are included in the model as per the methodology suggested by Balduzzi and Lynch (1999) and further developed in the work of DeMiguel, Garlappi and Uppal (2009) for the three minimum variance portfolios and benchmarks.

Let $\mathrm{Rp}$ denote the portfolio return before the revision, which is given by the formula:

$R p=\sum_{i=1}^{n} R i, t+1 * W i, t$

Portfolio rebalancing product at time $\mathrm{t}+1$ will be a trade with a magnitude of $|\mathrm{Wi}, \mathrm{t}+1-\mathrm{Wi}, \mathrm{t}|$. Let $\mathrm{c}$ denote the proportional transaction cost. After each revision period, the overall transaction cost will be:

$c * \sum_{i=1}^{n}|\mathrm{Wi}, \mathrm{t}+1-\mathrm{Wi}, \mathrm{t}|$

The Wealth net of transaction cost at time $t+1$ can be written as:

$$
\text { Wealth }_{t+1}=\text { Wealth }_{t} *(1+R p) *\left[1-c * \sum_{i=1}^{n}|\mathrm{Wi}, \mathrm{t}+1-\mathrm{Wi}, \mathrm{t}|\right]
$$

The Return net of transaction cost is given:

$R n=\frac{\text { Wealth }_{t+1}}{\text { Wealth }_{t}}-1$

This process is repeated every revision period for all portfolios and benchmarks. Portfolio rebalancing is applied every one and three years. Since benchmarks are often passive portfolio strategies with low management costs and low trading volumes, they are rebalanced every five years. Transaction costs applied are 50 basis points (bps) and 25 bps. The next section introduces the data used in this study.

\section{DATA}

The data used in this research are monthly and weekly returns of regional bond, equity indices and global oil, gold indices downloaded from DATASTREAM database software package available at ALBA Graduate Business School. The period under consideration is December 1996 - May 2013.

Table 1 lists the global asset portfolio indices. When identifying particular risk-return profiles for equity and bonds, the world is assumed to be fractioned in to four regions. Since more information is available, the bond market for Europe and the US is considered to be separate, corporate and government, to better understand which segment affects the performance behavior of GMVP and/or benchmarks. The table reports the indices used for constructing through optimization of the global minimum variance portfolio of the global assets that comprise stocks, bonds, gold and oil. The time series of monthly and weekly returns is taken into consideration for each index. The data considered cover the period December 1996 - May 2013 and the same data are used to construct benchmark number 2 - the equally weighted portfolio $(1 / \mathrm{N})$. The source is DATASTREAM database package available at ALBA Graduate Business School.

The value weighted benchmark of the global asset minimum variance portfolio is the one investing $60 \%$ in MSCI WORLD INDEX (equity global index) and 40\% in JPM GLOB AL BROAD INDEX (bond global index). 
Table 1: Global Asset Portfolio Constituents

\begin{tabular}{ll}
\hline CGBI WGBI EU ALL MATS & Europe Gov. Bond Index \\
REX GENERAL BOND & Europe Corp. Bond Index \\
CGBI USBIG OVERALL ASIA PACIFIC & Asia Pacific Bond Index \\
BARCLAYS EM WORLD ALL SERIES* & Emerging World Bond Index \\
ML CORP MASTER & US Corp. Bond Index \\
CGBI WGBI US ALL MATS & US Gov. Bond Index \\
MSCI EUROPE & European Stock Index \\
MSCI EM :I & Emerging World Stock Index \\
MSCI AC ASIA PACIFIC & Asia Pacific Stock Index \\
MSCI USA & US Stock Index \\
MLCX Gold & Gold Index \\
S\&P GSCI Crude Oil & Oil Index \\
\hline
\end{tabular}

*For Barclays Emerging Market World All Series bond index, DATASTREAM reports capital gains, but not total returns (including coupon). A total yearly return of 5\% split monthly was added for approximating the coupon rate. The modification refers to the capital gain/coupon rate features of the Barclays EM Bond Indices. On May 23, 2014 reports a Yield To Maturity (YTM) of approx. 5.8\% and capital gain of approximately 1\%, at Benchmark Index Returns - daily updates from Barclays. It is assumed that the current yield of the index has been constant throughout the period under consideration.

This is a common benchmark, referring to Hensel and Ziemba (1995) and Asness et al. (2012), used to assess investment performance in a portfolio with different asset classes. The second benchmark is the equally weighted portfolio constructed with the twelve indices included in Table 1. Since considered passive investment strategies, benchmarks are rebalanced every five years. All returns used in this research are EURO returns.

Next section describes the main empirical findings of this paper.

\section{EMPIRICAL ANALYSIS}

This section analyzes whether the use of weekly returns in GMVPs optimization reduces risk of minimum variance portfolios towards benchmarks and GMVPs estimated by monthly returns.

Table 2 reports the monthly optimization annualized first and second moments of returns results. The annualized maximum and minimum return are shown for the period under observation. The statistics are presented for the unconstrained, constrained and maximum constrained GMVPs of global asset portfolios and benchmarks, resulted from the portfolio construction approach described in the methodology section. The portfolio rebalancing/reinvestment period is one year. Monthly realized returns are net of transaction costs, which were assumed as 50 bps or 25 bps and applied as described in equation 8 .

Table 3 reports the weekly optimization annualized first and second moments of returns results. The annualized maximum and minimum return are shown for the period under observation. The statistics are presented for the unconstrained, constrained and maximum constrained GMVPs of global asset portfolios and benchmarks, resulting from the portfolio construction approach described in the methodology section. The portfolio rebalancing/reinvestment period is one year. Weekly realized returns are net of transaction costs, which were assumed as 50 bps or 25 bps and applied in optimization as described in equation 8 .

Considering the maximum/minimum annualized return of monthly GMVPs in Table 2 and weekly GMVPs in Table 3, the out-of-sample volatility of GMVPs estimated from higher frequency data seem to be higher. Minimum (maximum) values range from $-82.41 \%$ to $-104.6 \%$ (from $78.5 \%$ to $124.86 \%$ ) through all weekly minimum variance portfolios - more than twice the extreme values shown from monthly GMVPs.

Tables 2 and 3 show that only the most restrictive constrained weekly GMVPs of global asset reduce risk (0.33 percentage points) more than return ( 0.18 percentage points) as compared with respective monthly GMVPs when one-year revision frequency is imposed. This finding is not in line with Jagannathan and Ma (2003) who 
conclude that unconstraint GMVP estimated using higher frequency data, such as daily returns, result in lower outof-sample volatility.

Table 2: Summary Statistics Of Monthly GMVP And Benchmarks - One-Year Revision Frequency

\begin{tabular}{lcccc|cccc}
\hline & \multicolumn{4}{c}{ 50 bps Transaction Cost } & \multicolumn{3}{c}{ 25 bps Transaction Cost } \\
\hline & Mean & Std. Dev. & Max. & Min. & Mean & Std. Dev. & Max. & Min. \\
\hline Unconstrained GMVP & $5.15 \%$ & $2.66 \%$ & $35.14 \%$ & $-21.44 \%$ & $5.14 \%$ & $2.43 \%$ & $26.63 \%$ & $-21.44 \%$ \\
Constrained GMVP & $5.25 \%$ & $2.55 \%$ & $29.10 \%$ & $-21.05 \%$ & $5.25 \%$ & $2.53 \%$ & $25.91 \%$ & $-21.05 \%$ \\
Max. Constrained GMVP & $5.48 \%$ & $4.97 \%$ & $50.51 \%$ & $-39.72 \%$ & $5.48 \%$ & $4.96 \%$ & $50.51 \%$ & $-39.72 \%$ \\
Benchmark 1 & $3.39 \%$ & $7.87 \%$ & $64.52 \%$ & $-82.55 \%$ & $3.39 \%$ & $7.87 \%$ & $64.52 \%$ & $-82.55 \%$ \\
Benchmark 2 & $5.59 \%$ & $8.34 \%$ & $73.75 \%$ & $-92.73 \%$ & $5.59 \%$ & $8.34 \%$ & $73.75 \%$ & $-92.73 \%$ \\
\hline
\end{tabular}

Gosier et al. (2005) and Clarke et al.'s (2006) findings support the use of daily data versus monthly data for the portfolio variance-covariance matrix estimation when the objective is risk reduction. The results of this paper, however, show that only the most restrictive portfolio experiences benefits if more frequent data are used as optimization input.

A few remarks should be considered at this point. First, this work uses weekly returns as optimization input. Data characteristics, such as serial correlation or market microstructure features highly mentioned in the finance literature, are not part of this study. However, referring to Jagannathan and Ma (2003), after accounting for such effects, results do not change. Second, the number of inputs for weekly returns and monthly returns is similar in this research. The choice is justified because the estimation window for the weekly $(T=52)$ and monthly $(T=60)$ GMVPs is four times greater than the number of variables $(N=12)$ in the covariance matrix. Specifically, the numbers of parameters to be estimated is not of the same or higher orders of magnitude, which means that the matrix is not singular. Both cases may be problematic in terms of estimation error.

Table 3: Summary Statistics of Weekly GMVP and Benchmarks - One-Year Revision Frequency

\begin{tabular}{lcccc|cccc}
\hline & \multicolumn{3}{c}{$\mathbf{5 0}$ bps Transaction Cost } & \multicolumn{3}{c}{ 25 bps Transaction Cost } \\
\hline & Mean & Std. Dev. & Max. & Min. & Mean & Std. Dev. & Max. & Min. \\
\hline Unconstrained & & & & & & & & \\
GMVP & $4.66 \%$ & $3.08 \%$ & $124.84 \%$ & $-104.66 \%$ & $4.64 \%$ & $2.60 \%$ & $76.65 \%$ & $-75.43 \%$ \\
Constrained GMVP & $4.94 \%$ & $2.70 \%$ & $78.50 \%$ & $-82.41 \%$ & $4.94 \%$ & $2.69 \%$ & $78.50 \%$ & $-82.41 \%$ \\
Max. Constrained & $5.30 \%$ & $4.64 \%$ & $117.85 \%$ & $-98.88 \%$ & $5.30 \%$ & $4.64 \%$ & $117.85 \%$ & $-98.88 \%$ \\
GMVP & & & & & & & & \\
Benchmark 1 & $3.57 \%$ & $9.15 \%$ & $288.24 \%$ & $-301.02 \%$ & $3.57 \%$ & $9.15 \%$ & $288.24 \%$ & $-301.02 \%$ \\
Benchmark 2 & $5.21 \%$ & $8.21 \%$ & $206.59 \%$ & $-360.91 \%$ & $5.21 \%$ & $8.21 \%$ & $206.59 \%$ & $-360.91 \%$ \\
\hline
\end{tabular}

Referring to the findings of this paper, monthly GMVPs have experienced higher return compared to weekly GMVPs. This section concludes that GMVPs estimated from monthly returns dominate empirically GMVPs estimated from weekly returns, when rebalanced yearly, referring to the results in Tables 2 and 3 . The next section compares the out-of-sample risk reduction of weekly and monthly GMVP when revision frequency is applied every three years.

\section{Special Case: Three-Year Revision Frequency}

Table 4 reports the monthly optimization annualized first and second moments of returns results. The annualized maximum and minimum returns are shown for the period under observation. The statistics are presented for the unconstrained, constrained and maximum constrained GMVPs of global asset portfolios and benchmarks resulting from the portfolio construction approach described in the methodology section. The portfolio rebalancing/reinvestment period is one year. Monthly realized returns are net of transaction costs, which were assumed as 50 bps or 25 bps and applied as described in equation 8 .

Table 5 reports the weekly optimization annualized first and second moments of returns results. The annualized maximum and minimum return are shown for the period under observation. The statistics are presented for the unconstrained, constrained and maximum constrained GMVPs of global asset portfolios and benchmarks 
resulting from the portfolio construction approach described in the methodology section. Weekly realized returns are net of transaction costs, which were assumed 50 bps or 25 bps and applied in optimization as described in equation 8.

If portfolio rebalancing is done every three years in the optimization exercise, as shown in Tables 4 and 5, less frequent rebalancing weekly GMVP suffers an increase in out-of-sample fluctuation. The downside risks seem to substantially increase, with weekly GMVP rebalanced every three years experiencing minimum return, from $113.69 \%$ to $-232.69 \%$, more than $130 \%$ the downside potential of more frequently rebalanced weekly GMVPs.

Unconstrained weekly GMVPs perform better in terms of realized return as compared to monthly counterparts. Weekly GMVPs of global assets revised every three years show higher risk profile, as compared with monthly GMVPs, independently from the constraints imposed or transaction costs assumed. This result is consistent with Gosier et al. (2005) who suggest that the optimal estimation window should be at least four times higher than the holding period ${ }^{2}$.

Table 4: Summary Statistics Of Monthly GMVP And Benchmarks - Three-Year Revision Frequency

\begin{tabular}{lcccc|cccc}
\hline & \multicolumn{3}{c}{ 50 bps Transaction Cost } & \multicolumn{3}{c}{ 25 bps Transaction Cost } \\
\hline & Mean & Std. Dev. & Max. & Min. & Mean & Std. Dev. & Max. & Min. \\
\hline Unconstrained GMVP & $5.01 \%$ & $2.59 \%$ & $31.61 \%$ & $-28.39 \%$ & $5.00 \%$ & $2.36 \%$ & $26.63 \%$ & $-23.49 \%$ \\
Constrained GMVP & $5.07 \%$ & $2.51 \%$ & $29.10 \%$ & $-22.17 \%$ & $5.07 \%$ & $2.49 \%$ & $25.91 \%$ & $-22.17 \%$ \\
Max. Constrained GMVP & $5.82 \%$ & $4.92 \%$ & $50.51 \%$ & $-40.14 \%$ & $5.82 \%$ & $4.91 \%$ & $50.51 \%$ & $-40.14 \%$ \\
Benchmark 1 & $3.39 \%$ & $7.87 \%$ & $64.52 \%$ & $-82.55 \%$ & $3.39 \%$ & $7.87 \%$ & $64.52 \%$ & $-82.55 \%$ \\
Benchmark 2 & $5.59 \%$ & $8.34 \%$ & $73.75 \%$ & $-92.73 \%$ & $5.59 \%$ & $8.34 \%$ & $73.75 \%$ & $-92.73 \%$ \\
\hline
\end{tabular}

The results of this research differ from Zibri and Kukeli (2014) who find that unconstrained GMVPs generally offer lower variation out-of-sample. This paper finds that, only when one-year revision frequency is assumed and 25 bps transaction costs are imposed, unconstrained GMVPs estimated using weekly returns show lower out-of-sample variance, as compared with the other portfolios. Referring to the results of this paper, short-sale constrained weekly minimum variance portfolios offer lower out-of-sample variance than other weekly GMVPs.

Table 5: Summary Statistics of Weekly GMVP and Benchmarks - 3 Year Revision Frequency

\begin{tabular}{|c|c|c|c|c|c|c|c|c|}
\hline & \multicolumn{4}{|c|}{50 bps Transaction Cost } & \multicolumn{4}{|c|}{25 bps Transaction Cost } \\
\hline & Mean & Std. Dev. & Max. & Min. & Mean & Std. Dev. & Max. & Min. \\
\hline Unconstrained GMVP & $5.38 \%$ & $4.05 \%$ & $120.15 \%$ & $-232.69 \%$ & $5.37 \%$ & $3.96 \%$ & $120.15 \%$ & $-232.69 \%$ \\
\hline Constrained GMVP & $5.08 \%$ & $3.06 \%$ & $104.90 \%$ & $-113.53 \%$ & $5.08 \%$ & $3.06 \%$ & $104.90 \%$ & $-113.53 \%$ \\
\hline Max. Constrained GMVP & $5.34 \%$ & $5.03 \%$ & $124.65 \%$ & $-158.15 \%$ & $5.34 \%$ & $5.02 \%$ & $124.65 \%$ & $-158.15 \%$ \\
\hline Benchmark 1 & $3.57 \%$ & $9.15 \%$ & $288.24 \%$ & $-301.02 \%$ & $3.57 \%$ & $9.15 \%$ & $288.24 \%$ & $-301.02 \%$ \\
\hline Benchmark 2 & $5.21 \%$ & $8.21 \%$ & $206.59 \%$ & $-360.91 \%$ & $5.21 \%$ & $8.21 \%$ & $206.59 \%$ & $-360.91 \%$ \\
\hline
\end{tabular}

This section concludes that weekly GMVPs do not outperform monthly GMVPs in terms of out-of-sample risk reduction. The unique portfolio that results in lower out-of-sample variation, as compared with monthly GMVP, is upper/lower bound constrained weekly global minimum variance portfolio. Less frequent portfolio rebalancing increases the downside risk potential of weekly portfolios. The next section summarizes the conclusions of the research.

\section{CONCLUDING REMARKS}

This research studies the differences in performance of global minimum variance portfolios optimized using sample covariance matrix of weekly and monthly return. In this paper, the world is divided in four regions United States, Europe, Asia Pacific, and Developing countries. International indices of stocks, corporate/government bonds, oil and gold served monthly and weekly returns as input in the optimization exercise. GMVPs are optimized

\footnotetext{
${ }^{2}$ In the three-year revision frequency case, the estimation window of weekly GMVPs is one year (52 observations), while the holding period is three years (156 observations).
} 
considering assumptions of no imposed constraints and the minimum/maximum weight imposed constraints suggested by Jagannathan and Ma (2003).

The upper/lower bound constrained weekly GMVPs reduce risk more than return, as compared with monthly GMVPs, when one-year revision frequency is adopted. Weekly minimum variance portfolios, however, seem not to be as efficient in reducing out-of-sample variation of returns as monthly portfolios. The increase of rebalancing frequency weakens the risk reduction capacity of GMVPs estimated from higher frequency data based on the findings of this research.

To the best of the authors' knowledge, this is the first study that takes into consideration global asset classes to compare performance of a minimum variance portfolio constructed from weekly and monthly returns.

One of the extensions suggested for this study is the use of daily returns which, based on Jagannathan and Ma (2003), perform better when short sale is allowed. Moreover, the use of intraday return which, according to Liu (2009) may result in better performance than less frequent returns under specific conditions, may be applied for global assets minimum variance portfolios.

\section{ACKNOWLEDGEMENTS}

This research has been supported by ALBA Graduate Business School in Athens through the access to DATASTREAM. The authors thank Nikolaos Tessaromatis from EDHEC Business School and Athanasios Sakkas and Christos Cabolis from ALBA Graduate Business School for their great help and support with the data and academic feedback.

\section{AUTHOR INFORMATION}

Agim Kukeli has a Ph.D. (2004) in Economics, Colorado State University, Colorado, USA. Dr. Kukeli currently teaches economics at Black Hills State University, School of Business. He has been teaching economics and finance courses at both undergraduate and graduate levels at the Colorado Mesa University, Colorado State University, University of New York in Tirana, University of New York Prague, etc. His research interests are Monetary Theory and Policy, Foreign Direct Investment, Market Structure (Game theory), Financial Markets and Price Variability. Email: (agim.kukeli@bhsu.edu).

Arben Zibri is a Doctoral Candidate (2015, expected) in Economics at University "Aleksander Mojsiu", Durres, Albania. Arben Zibri is Senior Relationship Manager at Alpha Bank Albania and lecturer of finance at University of New York in Tirana and Epoka University, Albania. He has taught undergraduate and graduate courses, such as Portfolio Management, Corporate Finance, and Financial Institution and Markets. His research interests are Portfolio Theory, Capital Markets, Empirical Finance, Performance Analysis, and Market Risk.

Email: (arbenzibri@unyt.edu.al).

\section{REFERENCES}

Asness, C., Frazzini, A., \& Pedersen, L. (2012). Leverage Aversion and Risk Parity. Financial Analyst Journal, 68 (1), 47-59.

Balduzzi, P., \& Lynch, A. (1999). Transaction costs and predictability some utility cost calculations. Journal of Financial Economics, 52 (1), 47-78.

Behr, P., Guettler, A., \& Miebs, F. (2013, April). On portfolio optimization: Imposing the right constraints. Journal of Banking and Finance, 37(4), 1232-1242.

Behr, P., Guettler, A., \& Miebs, F. (2008, September). Is minimum-variance investing really worth the while? An analysis with robust performance inference. (Working Paper).

Berens, T., Wied, D., \& Ziggel, D. (2013, May). A completely automated optimization strategy for global minimumvariance portfolios based on a new test for structural breaks. Sonderforschungsbereich (SFB) 823, Discussion Paper No. 19. 
Chan, L. K. C., Karceski, J., \& Lakonishok, J. (1999, Winter). On portfolio optimization: Forecasting covariances and choosing the risk model. The Review of Financial Studies, 12(5), 937-974.

Chopra, K. V., \& Ziemba, T. W. (1993, Winter). The effect of errors in means, variances, and covariances on optimal portfolio choice. Journal of Portfolio Management, 19(2), 6-11.

Clarke, R., de Silva, H., \& Thorley, S. (2006, Fall). Minimum-variance portfolios in the U.S. equity market. The Journal of Portfolio Management, 33(1), 10-24.

Clarke, R., de Silva; H., \& Thorley, S. (2011, Winter). Minimum-variance portfolio composition. The Journal of Portfolio Management, 37(2), 31-45.

DeMiguel, V. Garlappi, L., \& Uppal, R. (2009, May). Optimal versus naïve diversification: How inefficient is the 1/n portfolio strategy? The Review of Financial Studies, 22(5), 1915-1953.

DeMiguel, V., Garlappi, L., Nogales J. F., \& Uppal, R. (2009, May). A generalized approach to portfolio optimization: Improving performance by constraining portfolio norms. Management Science, 55(5), 798812.

Fama, E., \& French, K. (1993, Feb.). Common risk factors in the returns on stocks and bonds. Journal of Financial Economics, 33(1), 3-56.

Frahm, G., \& Memmel, C. (2010, Dec.). Dominating estimators for the global minimum variance portfolios. Journal of Econometrics, 159(2), 289-302.

Frahm, G. (2010, Dec.). Linear statistical inference for global and local minimum variance portfolios. Springer Statistical Papers, 51(4), 789-812.

Gosier, K., Madhavan, A., Serbin, V., \& Yang, J. (2005). Toward Better Risk Forecasts. The Journal of Portfolio Management, 31(3), 82-91.

Healton, J., \& Lucas, D. (2000, June). Portfolio choice and asset prices: The importance of entrepreneurial risk. The Journal of Finance, 55(3), 1163-1198.

Hensel, Ch., \& Ziemba, W. (1995).United States Investment Returns during Democratic and Republican Administrations, 1928-1993. Financial Analyst Journal, 51 (2), 61-69.

Hollifield, B., \& Green, R. (1992, Dec.). When will mean-variance efficient portfolios be well diversified? The Journal of Finance, XLVII(5), 1785-1809

Kempf, A., \& Memmel, C. (2006, Oct.). Estimating the global minimum variance portfolio. Schmalenbach Business Review (SBR), 58, 332-348.

Kosowski, R., Narayan, N. Y., \& Teo, M. (2007, April). Do hedge funds deliver alpha? A bayesian and bootstrap analysis. Journal of Financial Economics, 84(1), 229-264.

Jagannathan, R., \& Ma, T. (2003, Aug.). Risk reduction in large portfolios: Why imposing the wrong constraints helps. The Journal of Finance, LVIII(4).

Ledoit, O., \& Wolf, M. (2003, Dec.). Improved estimation of the covariance matrix of stock returns with an application to portfolio selection. Journal of Empirical Finance, 10(5), 603-621.

Ledoit, O., \& Wolf, M. (2004, Summer). Honey, I shrunk the sample covariance matrix. The Journal of Portfolio Management, 30(4), 110-119.

Ledoit, O., \& Wolf, M. (2008, Dec.). Robust performance hypothesis testing with the Sharpe ratio. Journal of Empirical Finance, 15(5), 850-859.

Ledoit, O., \& Wolf, M. (2014, Jan.). Nonlinear Shrinkage of the Covariance Matrix for Portfolio Selection: Markowitz meets Goldilocks. Working Paper.

Liu, Q. (2009, June-July). On Portfolio Optimization: How and when do we benefit from high-frequency data?. Journal of Applied Econometrics, 24(4), 560-582.

Markowitz, H. (1952, March). Portfolio selection. The Journal of Finance, 7(1), 77-91.

Pantaleo, E., Tumminello, M., Lillo, F., \& Mantegna, N. R. (2011, July). When do improved covariance matrix estimators enhance portfolio optimization? An empirical comparative study of nine estimators. Quantitative Finance, 11(7), 1067-1080.

Roll, R. (1992, Summer). A mean/variance analysis of tracking error. The Journal of Portfolio Management, 18(4), 13-22.

Zibri, A., \& Kukeli, A. (2014, November/December). Does GMVP Strategy Reduce Risk? A Global Asset Approach. Journal of Applied Business Research, 30(6), 1863-1872. 